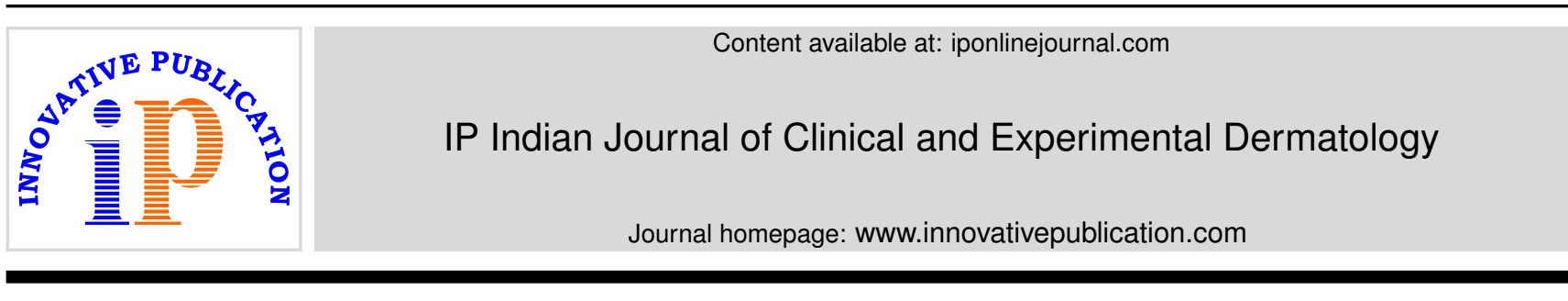

Original Research Article

\title{
Autoimmune associations of alopecia areata in pediatric population - A study in tertiary care centre
}

\author{
Sagar Nawani ${ }^{1}$, Teki Satyasri ${ }^{1, *}$, G. Narasimharao Netha1 ${ }^{1}$, G Rammohan', \\ Bhumesh Kumar ${ }^{1}$ \\ ${ }^{1}$ Dept. of Dermatology, Venereology \& Leprosy, Gandhi Medical College, Secunderabad, Telangana, India
}

\section{A R T I C L E I N F O}

\section{Article history:}

Received 21-01-2020

Accepted 24-02-2020

Available online 29-04-2020

Keywords:

Alopecia areata

Auto immunity

Pediatric population

\begin{abstract}
A B S T R A C T
Alopecia areata (AA) is second most common disease leading to non scarring alopecia . It occurs in many patterns and can occur on any hair bearing site of the body. Many factors like family history, autoimmune conditions and environment play a major role in its etio-pathogenesis. Histopathology shows bulbar lymphocytes surrounding either terminal hair or vellus hair resembling "swarm of bees" appearance depending on chronicity of alopecia areata. Alopecia areata in children is frequently seen. Pediatric AA has been associated with atopy, thyroid abnormalities and a positive family history. We have done a study to find out if there is any association between alopecia areata and other auto immune diseases in children. This study is an observational study conducted in 100 children with AA to determine any associated autoimmune conditions in them. SALT score helps to assess severity of alopecia areata. Severity of alopecia areata was assessed by SALT score-1. S1- less than $25 \%$ of hairloss, 2. S2- $25-49 \%$ of hairloss, 3. 3.S3- 50-74\% of hairloss.
\end{abstract}

(C) 2020 Published by Innovative Publication. This is an open access article under the CC BY-NC-ND license (https://creativecommons.org/licenses/by/4.0/)

\section{Introduction}

Alopecia areata (AA) is a common chronic, multifactorial disorder with autoimmunity in etio-pathogenesis leading to non cicatricial alopecia. It can present either as patchy hair loss or as diffuse pattern without any clinical inflammatory changes. Patchy pattern is most common type. It can affect any hair bearing site of the body. ${ }^{1}$ The term Alopecia areata was coined by Sauvages in $1760 .^{2}$ It was first described by Cornelius Celsus . It accounts for $0.7 \%$ in India. ${ }^{3}$ It is the most frequent non cicatricial alopecia, after male and female pattern baldness. Both males and females are equally affected, but few population surveys have shown male preponderance. ${ }^{4}$ It can occur at any age. $20 \%$ of cases occur in children and $60 \%$ of AA patients can have their first patch before second decade. Family history of $8.7-20 \%$ was detected in these cases. Often, they are noticed either by hairdresser or family member. It may present as single or

\footnotetext{
* Corresponding author.

E-mail address: satyadermatologist@gmail.com (T. Satyasri).
}

multiple patches or suddenly can become grey overnight. Small patches coalasce with each other and form larger patches [Figures 1 and 2].

There are many types of alopecia areata.

1. Patchy alopecia areata: This is most common form. One or more patches of hair loss on scalp is present.

2. Alopecia totalis: whole scalp is devoid of hair.

3. Alopecia universalis: total or near total loss of whole body hair.

4. Ophiasis: hair loss in a band like fashion around the circumference of the head.

5. Sisaphio: This is the reverse of ophiasis wherein hair is lost on the top of the head, sparing the sides lower back of the head (hence the term is just spelled backwards).

6. Alopecia incognito: diffuse total hairloss with positive pull test, yellow dots, miniaturized short regrowing hair, but without nail involvement.

7. Canities subita: other wise called Marie Antoinette syndrome: characterized by sudden "overnight" greying of hair in which pigmented hair is lost. 
Autoimmunity is the most common cause. Alopecia areata usually occurs in association with autoimmune diseases like Hashimoto's thyroiditis, addison's disease, Pernicious anaemia, Diabetes mellitus, atopic dermatitis, Ipex syndrome, crohn's disease, lupus erythematosus. ${ }^{5,6}$

\section{Objective}

This study is an observational study conducted in 100 children with AA to determine their clinical patterns \& any associated autoimmune conditions associated in them.

\section{Material and Methods}

This study was conducted in 100 children (0-18 years) with alopecia areata for a period of 9 months in OPD at Gandhi hospital/Gandhi medical college, Secunderabad. Diagnosis in all cases was based on history of patchy abrupt loss of hair, with or without progression on absolutely normal looking skin. Patients were thoroughly examined and evaluated for associated autoimmune conditions. During examination, the size of patches, their number \& into which pattern it fits were recorded. Age at onset, duration of disease, progression, symptoms of atopy such as nasal symptoms, sneezing, red and watery eyes and history of asthma were noted in detailed proforma. Investigations like complete blood picture, thyroid profile, cortisol levels, peripheral smear, random blood sugar were performed. Severity of alopecia areata was calculated by SALT score. ${ }^{7}$

\section{Results}

1. Among 100 patients of alopecia areata, 68 were males and 32 were females[Table 1]

2. Autoimmune association was seen in 43 patients.

3. Of the autoimmune diseases associated with Alopecia areata, hypothyroidism and atopy showed the highest frequency. Out of 32 females, 17 had hypothyroidism and 10 had atopy and among 68 males, 12 had atopy and 4 had hypothyroidism[Table 2]

4. In total, 21 patients were detected with Hypothyroidism and 22 had atopy.

5. No other autoimmune disease was noted.

\section{Discussion}

Alopecia areata is estimated to occur in $0.1-0.2$ percent of general population at some point during their life time. Etiology includes genetic factors, autoimmunity and environmental factors and also emotional stress. Genetic etiology was found to have significance in $10-20 \%$ in various different studies. HLA studies have shown that HLA-A1, HLA-DQ1 and HLA-DQ3 were associated in more cases of alopecia areata than controls. HLA-DR 16 was significantly less common in patients with alopecia areata than controls concluding that this allele might

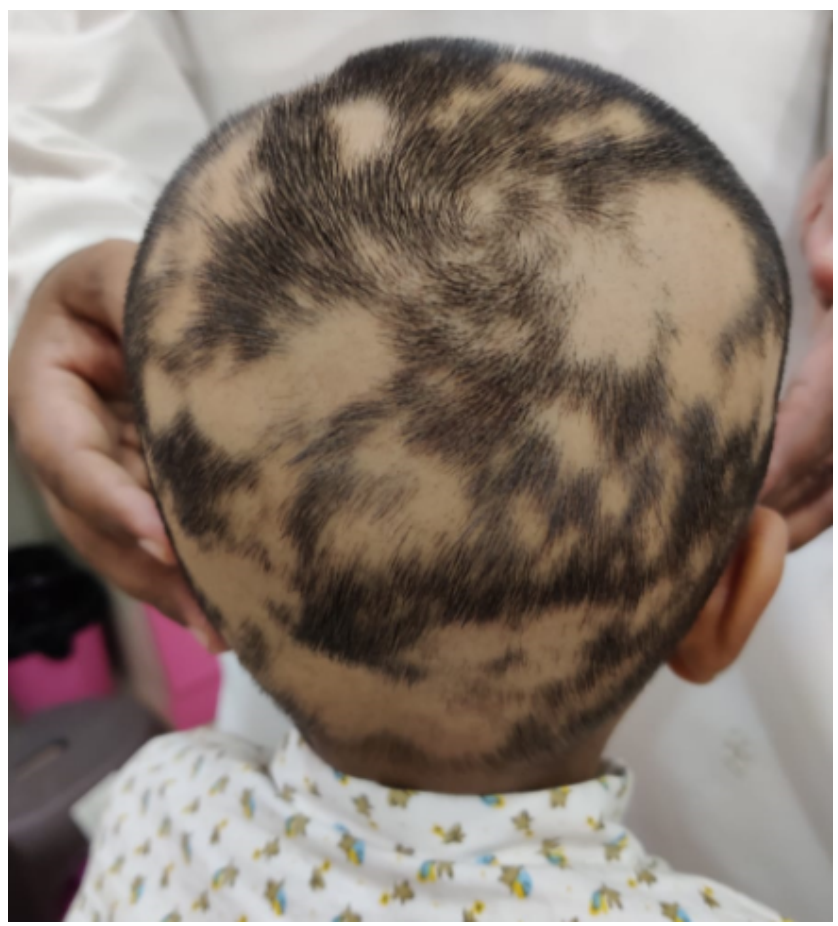

Fig. 1:

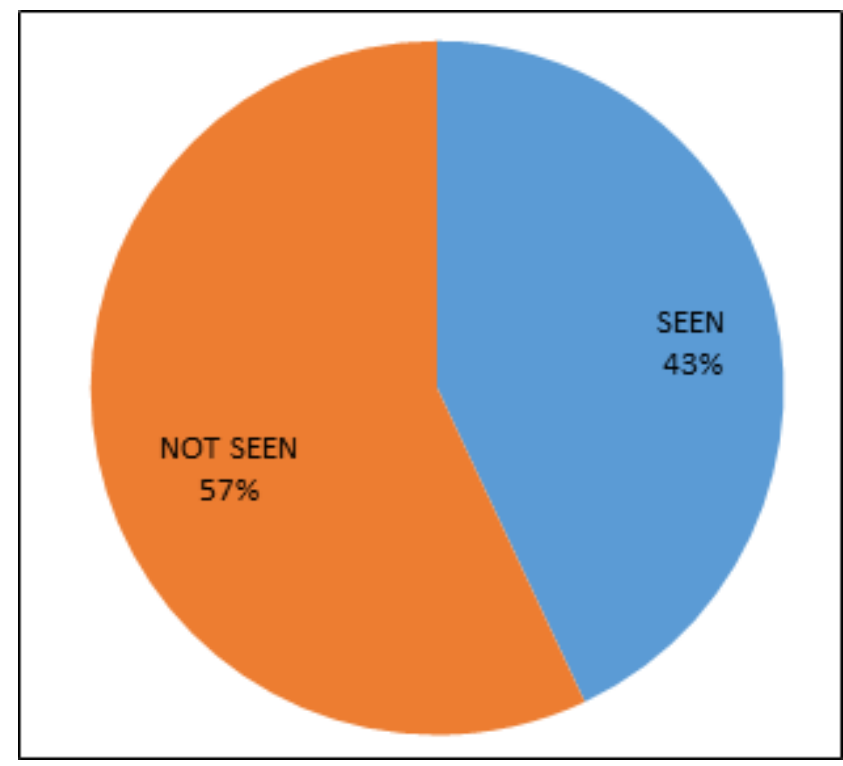

Fig. 2: Showing autoimmune association in our study 
Table 1: Showing sexpredilection

\begin{tabular}{lll}
\hline Female & Males & Total \\
32 & 68 & 100 \\
\hline
\end{tabular}

Table 2: Showing no. of casesof alopecia areata with atopy and hypothyroidism

\begin{tabular}{llll}
\hline Gender & Associated Disease & & Typothyroidism \\
Male & Atopy & 4 & 16 \\
Female & 12 & 17 & 27 \\
Total & 10 & 21 & 43 \\
\hline
\end{tabular}

be having protective role for alopecia areata ${ }^{8}$ Emotional stress along with atopic state has been implicated in etio-pathogenesis of alopecia areata. ${ }^{9}$ One of the main systemic associations of autoimmune disease is with thyroid abnormalities. The incidence of thyroid disease varied from $8-28 \%$ in patients with AA. In our study, we found hypothyroidism in $21 \%$ of cases. although the effects of hypothyroidism on hair is known from long.., its exact mechanism is not known. Delay in anagen growth phase that occurs in hypothyroidism may be one of the main cause. Other associated autoimmune diseases like vitiligo, pernicious anemia, lupus erythematosus, ulcerative colitis reported by many authors were not found in our study. Autoimmunity -T-cell response against unknown follicular self antigen. ${ }^{10}$ Now, focus has been given to follicular melanocytes. Patients presenting with AA in childhood $(<10$ yrs of age) either can have associated atopic dermatitis or SLE \& those patients who developed alopecia areata in between 10-20 years have greater chances of psoriasis or rheumatoid arthritis in one study but in our study, we did not encounter such patients.

Atopy has been reported to occur with an increased frequency in patients with alopecia areata. In Ikeda's classification of alopecia areata of 1989 patients, atopy constituted $10 \%$ of cases. In a study by Muller and Winkelmann out of 736 patients, $11 \%$ are either associated with asthma or atopic dermatitis. In our study, atopy was associated in $22 \%$ patients with alopecia areata.

SALT score is useful to find out the quantitative assessment of scalp hair loss. The entire scalp was divided into 4 parts based on the surface area, top (40\% - 0.4), posterior $(24 \%-0.24)$, right side $(18 \%-0.18)$, and left side of scalp (18\% - 0.18). Percentage of hair loss in each area is determined independently and is multiplied by the percentage of scalp covered in that area of the scalp, and summing the products of each area will give the SALT score respectively, then the SALT score can be calculated. SALT score is easily reproducible and validated. However, it does not include hair pigmentation, body hair, and nail involvement.

\section{Conclusion}

In our study, we conclude that
1. Males are more affected than females

2. Autoimmune association was more common in females than in males.

3. Most common associations being hypothyroidism and atopy in $43 \%$ of patients[pie chart].

4. We did not find any association with diabetes mellitus, SLE, psoriasis or Rheumatoid arthritis.

5. These observations lead us to understand the importance of screening for thyroid profile and history of atopy in patients with alopecia areata especially in children.

\section{Source of funding}

None.

\section{Conflict of interest}

None.

\section{References}

1. Hordinsky M, Ericson M. Autoimmunity: Alopecia Areata. Journal of Investigative Dermatology Symposium Proceedings. 2004;9(1):73-78. Available from: https://dx.do1.org/10.1111/.1087-0024.2004.00835. 凶. doi:10.1111/.1087-0024.2004.00835.X.

2. Sharma VK, Dawn G, Kumar B. Profile of Alopecia areata in Northern India. Int J Dermatol. 1996;35:22-29.

3. Safavi KH, Muller SA, Suman VJ, Moshell AN, Ljrd M. Incidence of Alopecia areata in Olmsted County, Minnesota,1975 through 1989. Mayo Clin Proc. 1995;70:628-633.

4. Tan E, Tay YK, Goh CL, Giam YC. The pattern of Alopecia areata in Singapore-A study of 219. Asians Int J Dermatol. 2002;41:748-753.

5. Brenner R. Coincidences of alopecia areata, Vitiligo, Onychodystrophy, localized scleroderma and lichen planus. Dermatologica. 1979;159:356-358

6. Muller SA, Winkelmann RK. Alopecia areata. Arch Dermatol. 1963;88:290-297.

7. Olsen EA, Hordinsky MK, Price VH, Roberts JL, Shapiro J, Canfield D. National Alopecia Areata Foundation. Alopecia areata investigational assessment guidelines. Part II. National Alopecia Areata Foundatin. J Am Acad Dermatol. 2004;51:440-447.

8. Kavak A, Baykal C, Ozarmagan G, Akar U. HLA in alopecia areata. International Journal of Dermatology. 2000;39(8):589-592. Available from: https://dx.do1.org/10.1046/].1365-4362.2000.00921. ه. do1:10.1046/].1365-4362.2000.00921.X.

9. Manzoor S, Masood C. Alopecia areata in Kashmir: A study of 200 patients. Indian J Dermatol Venereol Leprol. 2001;67:324-329. 
10. Tobin DJ, Orentreich N, Bystryn JC, Fenton DA. Antibodies to Hair Follicles in Alopecia Areata. Journal of Investigative Dermatology. 1994;102(5):721-724. Available from: https://dx.doi.org/10.1111 1523-1747.ep12375477, doi:10.1111/1523-1747.ep12375477.

\section{Author biography}

Sagar Nawani Post Graduate

Teki Satyasri Associate Professor
G. Narasimharao Netha Professor and HOD

G Rammohan Associate Professor

Bhumesh Kumar Associate Professor

Cite this article: Nawani S, Satyasri T, Netha GN, Rammohan G, Kumar B. Autoimmune associations of alopecia areata in pediatric population - A study in tertiary care centre. IP Indian J Clin Exp

Dermatol 2020;6(1):41-44. 